\title{
Intervenções de enfermagem que capacitam a Pessoa em Situação Crítica: Revisão integrativa da literatura
}

\author{
Suse Antunes ${ }^{1}$, Anabela Mendes ${ }^{2}$ e Elisabete Silva ${ }^{3}$ \\ 1 Serviço de Medicina Intensiva do Hospital Fernando da Fonseca, Enfermeira. Estudante \\ do Curso de Mestrado em Enfermagem na Área de Especialização à Pessoa em Situação \\ Crítica, Escola Superior de Enfermagem de Lisboa, Portugal | \\ suseisabelantunes@gmail.com | https://orcid.org/0000-0000-0000-0000 \\ ${ }^{2}$ Departamento de Enfermagem Médico-Cirúrgica Adulto-Idoso. Professora Coordenadora. \\ Escola Superior de Enfermagem de Lisboa, Portugal | anabelapmendes@esel.pt | \\ https://orcid.org/0000-0002-0001-4862 \\ ${ }^{3}$ Unidade de Queimados do Hospital São José, Enfermeira - CHULC. Estudante do Curso \\ de Mestrado em Enfermagem na Área de Especialização Pessoa em Situação Crítica, \\ Escola Superior de Enfermagem de Lisboa, Portugal | mariabeta@sapo.pt | \\ https://orcid.org/0000-0000-0000-0000
}

Resumo: A Pessoa em Situação Crítica (PSC) é, por definição, alguém dependente de cuidados intensivos e tecnológicos. Os enfermeiros constatam que as pessoas que vivem este processo de transição, perdem capacidades relevantes, como por exemplo a de autocuidado e de tomada de decisão. Este trabalho tem como principais objetivos: (1) Identificar os fatores que influenciam a capacitação da PSC e (2) Identificar as Intervenções de enfermagem que capacitam a PSC. Realizou-se uma revisão integrativa da literatura, com pesquisa de artigos nas bases de dados MEDLINE e CINAHL e literatura cinzenta. Emergiram, aplicados os critérios de inclusão e exclusão, 8 artigos para análise e discussão. Identificou-se um conjunto de fatores que pela sua relevância influenciam a capacitação da PSC. Como Intervenções de Enfermagem que capacitam a PSC elencam-se: ajudar a pessoa a aceitar a dependência no autocuidado e a gerir expectativas; potenciar a confiança e motivação pela individualização dos cuidados e a gestão de sintomas; promover uma comunicação eficaz com pessoa doente e família, ao longo do processo de transição; subsidiar a gestão dos recursos disponíveis (pessoais, sociais e comunitários), que possibilitam o retorno ao quotidiano; e assumir a família como parceira nos cuidados. Concluise que as intervenções terapêuticas de enfermagem que capacitam a PSC, se enquadram num cuidado centrado no cliente, PSC e família.

Palavras-chave: Capacitar; Pessoa em Situação Critica; Intervenção Terapêutica de Enfermagem; Transição Saúde-Doença.

\section{Nursing Interventions to Empower the Person in Critical Situation: Integrative Literature} Review

Abstract: The Person in Critical Situation (PCS) is, by definition, someone dependent on intensive and technological care. Nurses find that people who live in this transition process lose relevant abilities, such as self-care and decision-making. This work has as main objectives: (1) To identify the factors that influence the training of the person in critical situation and (2) To identify the nursing interventions that empower the PCS. An integrative literature review was conducted, with research of articles in MEDLINE and CINAHL databases and grey literature. Once applied the inclusion and exclusion criteria, eight articles for analysis and discussion emerged. A set of factors that due to their relevance influence the empowerment of the PCS was identified. The Nursing Interventions that empower the PCS are: to help the person to accept dependence on self-care and to manage expectations; enhance confidence and motivation for individualization of care and symptom management; promote effective communication with sick people and family throughout the transition process; subsidize the management of available resources (personal, social and community), which enable the return to everyday life; and assume the family as a partner in care. It is concluded that the nursing therapeutic interventions that empower the PCS, are part of a centered-care on the client, person in critical situation and family.

Keywords: Empowerment; Critical Care; Critical Care Nursing; Health Transition. 


\section{Introdução}

A Pessoa em Situação Crítica (PSC), segundo Benner, Kyriakidis, \& Stannard (2011), é aquela que fica gravemente doente ou ferida e incapaz de manter, de forma independente, a estabilidade fisiológica ou está em alto risco de desenvolver rapidamente instabilidade fisiológica, sendo tipicamente dependente de cuidados intensivos contínuos e da tecnologia de apoio.

De acordo com Meleis (2010), os enfermeiros vivenciam momentos de transição com as pessoas, quando relacionados com a saúde, o bem-estar e a capacidade para o autocuidado, surgindo assim o conceito de Intervenção Terapêutica de Enfermagem. Esta caracteriza-se pela ajuda/suporte às pessoas, famílias e comunidades para lidarem com transições, antecipando respostas, fornecendo orientação, gerindo sintomas, melhorando a saúde e o bem-estar e apoiando o desenvolvimento de ações de autocuidado. Meleis (2018) concretiza que, embora os enfermeiros em contexto de UCl possam centrar a sua abordagem na doença, verifica-se a sua preocupação com a segurança dos doentes, o bem-estar, a promoção da saúde, a manutenção de hábitos saudáveis e o apoio para o regresso à maior normalidade possível na vida quotidiana.

$\mathrm{Na}$ interação, e da interação constante, os enfermeiros têm como foco a capacitação, considerando que está intimamente relacionada com o exercício de autonomia e autocuidado. Importa compreender e elencar quais as intervenções terapêuticas de enfermagem que capacitam a PSC.

É evidente a proximidade entre os conceitos de capacitação, de autocuidado e de autonomia. Mamashli et al. (2019) aferem que quando a capacitação, e a adesão ao tratamento são a maior prioridade nos sistemas de saúde, a estadia da pessoa doente no hospital é reduzida. Os enfermeiros desempenham um papel importante na melhoria do conhecimento e capacitação das pessoas. Segundo Funnell (2016), os objetivos dos cuidados com foco na capacitação e educação da PSC são: aumentar a autonomia, permitir a tomada de decisão informada, possibilitar a resolução de problemas e alcançar objetivos comportamentais auto-determinados. Para Jones et al. (2020), a capacidade é relacionada com a aptidão de uma pessoa para tomar decisões num determinado momento, ainda que com perda temporária de capacidade ou capacidade flutuante em outros momentos. Assim, a avaliação da capacidade mental é um aspeto fundamental e deve ser conduzida como parte de uma avaliação holística da condição e das necessidades das pessoas em ambientes de cuidados críticos, pela equipa multidisciplinar.

No que concerne ao envolvimento da família, Jones et al. (2020) referem que esta desempenha um papel permanente nos processos de transição, fornecendo informações valiosas sobre definições do que constituía a capacidade "normal" para a pessoa doente. Importa, ainda, compreender o impacto que a situação critica de um familiar tem para a família. Mendes $(2018$, p. 203) refere que "a vulnerabilidade experienciada (...) fragiliza no presente (...) e pode condicionar o futuro individual e coletivo [uma vez que a situação crítica da pessoa] (...) emerge de modo súbito na vivência quotidiana da família e está associada a qualquer das fases do seu ciclo de vida."

Segundo Meleis (2018), a mudança experienciada, nomeadamente de saúde-doença, que se revela na alteração das capacidades da pessoa, traduz uma transição. Este processo implica que a pessoa incorpore novos conhecimentos e altere ou reposicione tipologias de comportamento. Constata-se assim que as transições se caracterizam por diferentes etapas dinâmicas, marcos e pontos de viragem e podem ser definidas através de processos e/ou resultados a atingir (Meleis, 2010).

Willis et al. (2008) afirmam que as intervenções de enfermagem, através do desenvolvimento e a aplicação do conhecimento de enfermagem na prática clinica, podem ser facilitadoras, capacitando os seres humanos na visão e aquisição da saúde. promovendo a humanização, o significado, a tomada de decisão e a qualidade de vida. 
Considerando a transição saúde-doença, experienciada pela PSC, esta revisão integrativa da literatura, tem como objetivo: Identificar os fatores que influenciam a capacitação da PSC e Identificar as Intervenções de enfermagem que capacitam a PSC.

\section{Metodologia}

Considerados os objetivos da pesquisa, a elaboração da questão de investigação e definição dos critérios de seleção e inclusão de artigos foi suportada no método PICo (Quadro 1), baseado nas orientações do Joanna Briggs Institute for Evidence Based Practice (Joanna Briggs Institute, 2020).

Quadro 1. Critérios de Seleção e Critérios de Inclusão dos Artigos

\begin{tabular}{ll}
\hline Critérios de Seleção & Critérios de Inclusão \\
\hline Tipo de Participantes & $\begin{array}{l}\text { Pessoa adulta ou idosa em situação crítica } \\
\text { (idade igual ou superior a 18 anos) }\end{array}$ \\
$\mathbf{P}$ (Person/problema) - Pessoa adulta & $\begin{array}{l}\text { Intervenções terapêuticas de enfermagem } \\
\text { que capacitam a pessoa em situação crítica }\end{array}$ \\
$\begin{array}{l}\text { I (Intervention) - Intervenções terapêuticas } \\
\text { de enfermagem que capacitam }\end{array}$ & Estar em situação crítica \\
$\begin{array}{l}\text { Contexto } \\
\text { Co }(\text { Context }) \text { - Situação crítica }\end{array}$ & \\
\hline
\end{tabular}

A questão de investigação que emerge é: Quais as intervenções terapêuticas de enfermagem que capacitam a pessoa em situação crítica?

A pesquisa foi efetuada no universo temporal de 2010 a 31 de outubro de 2020 nas bases de dados MEDLINE e CINAHL. Foram ainda considerados outros estudos provenientes de literatura cinzenta, identificados no Google Scholar.

Foram utilizados os seguintes descritores, com as respetivas combinações: S1 Empowerment OR Patient Participation AND S2 - Critical care OR Critical care nursing OR Critical care Outcomes OR Critical Illness.

A estratégia de pesquisa utilizada incluiu todas as tipologias de artigos. Foram pesquisadas referências nos idiomas português, inglês e espanhol.

Foram aplicados os seguintes critérios de exclusão: população pediátrica (ou seja, idade inferior a 18 anos); artigos que não se direcionam à capacitação da pessoa doente; artigos que não mencionem pelo menos um de três pontos - (1) intervenções de enfermagem que capacitam; (2) fatores facilitadores da capacitação, (3) fatores inibidores da capacitação.

No diagrama apresentado na Figura 1, está representado o processo de seleção dos artigos. Na pesquisa inicial foram obtidos 58 artigos (nas bases de dados MEDLINE e CINAHL) e 7 artigos na literatura cinzenta, perfazendo um total de 65 artigos. Foram excluídos 5 artigos repetidos. Foram excluídas 33 referências após analise dos títulos, tendo em conta os critérios de inclusão e exclusão. Foram analisados os resumos de 28 artigos, e excluídos 10 artigos por se verificar que não se direcionavam ao objeto de estudo, nomeadamente a capacitação da PSC. Dos 18 textos completos que se pretendiam analisar, foram excluídos 10 artigos, sendo que a maioria foi por não haver acesso ao artigo integral, e outras referências por se verificar que, no texto em si, não abordavam intervenções de enfermagem que capacitam a PSC, nem fatores facilitadores ou inibidores da capacitação.

A apreciação dos artigos foi efetuada, de forma independente, pelo investigador principal. Os outros dois investigadores realizaram a revisão dos artigos e da respetiva análise. 


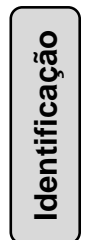

Artigos identificadas em base de dados eletrónicas (MEDLINE e CINAHL)

$$
(n=58)
$$

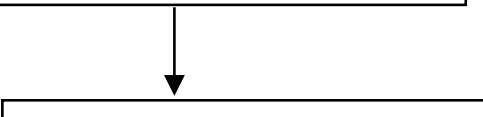

Artigos após a exclusão de artigos repetidos $(n=61)$

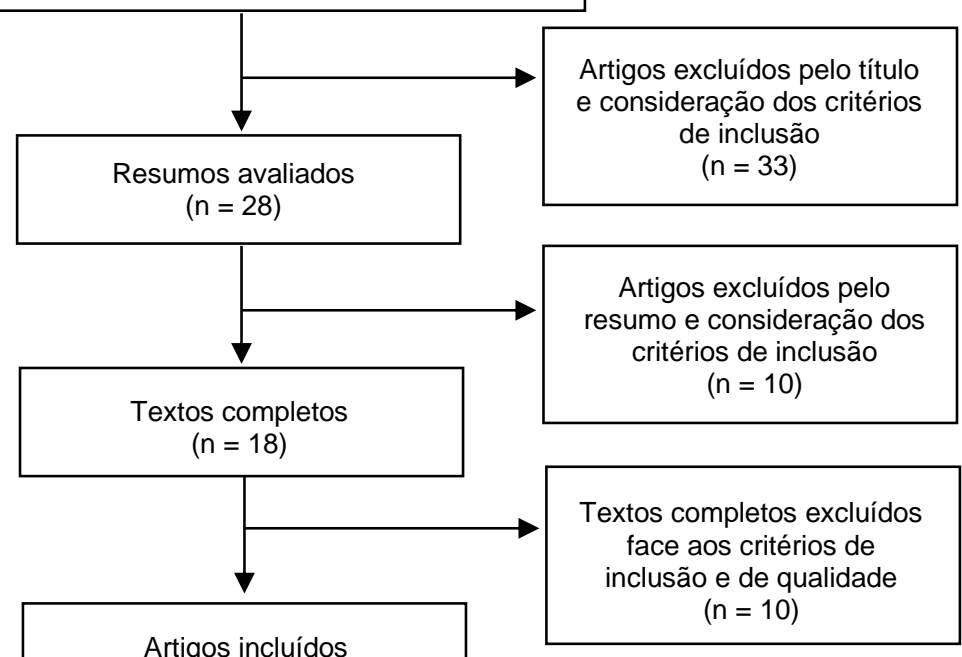

Fontes adicionais de artigos em literatura cinzenta $(\mathrm{n}=7)$
Artigos incluídos $(\mathrm{n}=8)$

Fig. 1. Diagrama de Seleção dos Artigos (adaptado de Prisma 2009 Flow Diagram).

\section{Resultados e Discussão}

O resultado final de apenas 8 artigos, demonstra que esta questão é, ainda, pouco explorada. Verifica-se que uma parte significativa dos artigos excluídos, nas várias etapas do processo, abordam a capacitação num contexto que não o de situação critica.

Verifica-se ainda na análise que a maior parte destes artigos procura e dá resposta aos fatores que facilitam ou inibem a capacitação da pessoa em situação critica, mas não se debruçam especificamente sobre as intervenções de enfermagem que capacitam a PSC. Foram identificadas duas grandes categorias: (1) Fatores inibidores da capacitação da PSC e (2) Fatores facilitadores da capacitação da PSC. Tendo por base os dados produzidos pela RIL, organizados nas referidas categorias pretende-se elencar as intervenções de enfermagem que influenciam a capacitação da PSC.

Os 8 artigos selecionados para análise, enquadram-se num paradigma qualitativo, o que motivou a apresentação dos resultados e conclusões através do método de síntese narrativa.

\subsection{Fatores Inibidores da Capacitação da PSC}

A condição de estar em situação critica, quando percecionada pela pessoa doente, é geradora de enorme vulnerabilidade pela dependência experienciada. Emergem aspetos significativos que a PSC relaciona com a dependência, nomeadamente: a sensação de impotência, limitações físicas, a dependência da tecnologia (associada a estarem conectadas a ventilação mecânica invasiva) e incapacidade para o autocuidado (Lindberg et al., 2015; Lykkegaard \& Delmar, 2015). As pessoas descrevem que a dependência dos profissionais de saúde foi de tal forma marcante, que a sensação de impotência perdurou mesmo após transferência para a enfermaria (Major et al., 2019). 
Algumas pessoas relatam que a condição de situação critica em que se encontram cria, por si só, alguma passividade. Aceitam que sejam realizados todos os procedimentos necessários, uma vez que confiam nos conhecimentos dos profissionais de saúde e assumem que o seu potencial de intervenção é diminuto. Aceitam a perda do controlo e a dependência como forma de lidar com a sua nova condição de vulnerabilidade, por considerarem agradável e securizante que todas as suas necessidades sejam satisfeitas. (Lindberg et al., 2015; Lykkegaard \& Delmar, 2015; Wåhlin et al., 2017)

Intimamente relacionado com a dependência, surge o segundo fator inibidor da capacitação da PSC: a ausência de individualização nos cuidados.

Algumas situações mais significativas estão relacionadas com a falta de privacidade. É descrito pelas pessoas doentes que se sentiam expostas durante os cuidados de higiene e conforto. A exposição do seu corpo e a forma como os profissionais se posicionam no cuidado ao seu corpo, revelam-se determinantes e significativas, pela positiva ou pela negativa. Outro aspeto determinante era o desinteresse dos profissionais de saúde, percecionado pela pessoa doente, relativamente a si. Este revela-se no modo como a consideravam, ou não, na interação. As situações em que os profissionais "falavam entre si", ignorando a presença da pessoa doente, eram vividas negativamente, desencadeando ansiedade e sofrimento para a PSC (Lindberg et al., 2015; Lykkegaard \& Delmar, 2015; Wahlin, Samuelsson \& Agren, 2017).

$\mathrm{Na}$ relação enfermeiro-PSC são conotadas como negativas as situações em que o enfermeiro tem um registo de interação que se caracteriza pela superficialidade, traduzido pelo reduzido contacto visual e ausência de empatia. (Lindberg et al., 2015; Lykkegaard \& Delmar, 2015; Wahlin, Samuelsson \& Agren, 2017). Este tipo de atitudes, para além de condicionarem a individualização dos cuidados, inviabilizam a interação e traduzem uma comunicação ineficaz.

Major et al. (2019) consideram que a falta de comunicação, ou comunicação ineficaz, com a PSC e família, em registo escrito para posterior continuidade dos cuidados, contribui para a perceção da PSC ser negligenciada. Exemplos disso são as cartas de alta/ transferência com informação incompleta ou insuficiente, que geram cuidados inadequados após a alta ou transferência da PSC.

Mesmo quando as informações prestadas são claras, verifica-se que estas não são suficientes, por si só, para a capacitação da PSC, uma vez que há alguns fatores a considerar. E necessário englobar a qualidade das informações e o contexto em que estas são fornecidas, para um aumento no grau de capacitação da PSC. Algumas pessoas doentes relatam que as informações transmitidas ao longo do seu internamento foram insuficientes, por não particularizarem o que tinha ocorrido e qual o motivo de internamento na UCl; o porquê de não conseguirem falar, comer ou beber, etc (Wahlin, Samuelsson \& Agren, 2017; Wåhlin, 2017).

No que diz respeito à participação da família nas intervenções, esta engloba inúmeros benefícios para uma transição saúde-doença facilitada. Benner, Kyriakidis, \& Stannard (2011) salientam que, para assegurar a compreensão de conteúdos pela família, importa deter um determinado grau de competência em comunicação, compreensão, paciência e escuta ativa. Ainda assim, verifica-se a incerteza sobre aspetos do âmbito organizacional, nomeadamente, as políticas existentes na $\mathrm{UCl}$ em relação à participação da família e até mesmo sobre a possibilidade de visita e em que moldes. Há uma perceção de que as famílias não são bem-vindas, nem são participantes, devido ao impedimento para a família estar presente em determinados momentos de intervenções à PSC (Hardin, 2012).

No atual contexto pandémico, verifica-se o abandono de estratégias já criadas e implementadas para o envolvimento da família da PSC, pois as famílias raramente estão presentes no hospital e quase nunca é permitida a presença junto da PSC. Estas condicionantes, levaram alguns familiares a manifestarem sensação de frustração, tristeza e pesar por não ser possível estarem presentes ao lado dos seus entes queridos (Devlin et al., 2020). Desta forma, assume-se a restrição de visitas (e/ou não participação da família) como um inibidor da capacitação da PSC. 


\subsection{Fatores Facilitadores da Capacitação da PSC}

O primeiro fator que facilita a capacitação da PSC é a aceitação da condição de dependência e a gestão de expetativas, sendo uma base fundamentada para o sucesso dos fatores subsequentes.

Importa ressalvar que, a PSC pode encontrar-se em diversos estados de autonomia relativos a múltiplos aspetos, os quais se podem sobrepor, havendo diversos graus de autonomia para diferentes aspetos. No processo de recuperação, pode haver recuperação de algumas capacidades, mas não de outras (Lindberg et al., 2015).

Segundo Devlin et al. (2020), numa fase em que a PSC se encontra profundamente sedada, e em que há restrições nas visitas de familiares e profissionais de áreas mais variadas de atuação (como sucede no contexto pandémico), é da responsabilidade dos profissionais de saúde que contactam com a PSC a operacionalização de metas diárias para a reabilitação e recuperação da mobilidade.

A PSC vivencia os aspetos tecnológicos como normais e como fonte de segurança, sendo inegociável a sua utilização num contexto de necessidade de cuidados médicos avançados. As pessoas doentes referem que, em determinada fase, é positivo o fator de dependência e rendem-se aos cuidados, não querendo interferir nos procedimentos que Ihes podem salvar a vida, tendo confiança nos profissionais pela sua experiência e conhecimento (Lindberg et al., 2015).

A gestão de expectativas é valorizada pela PSC, como facilitadora para uma experiência de transição positiva. $\mathrm{O}$ ajuste à nova realidade, com conhecimento que a recuperação pode ser demorada, constitui um catalisador do início do processo de cura, estabelecendo metas realistas de reabilitação e preparando para a longa e difícil caminhada para a recuperação (Major et al., 2019).

O processo de capacitação não está unicamente relacionado com aspetos físicos. A PSC deve ter um papel ativo na sua recuperação, construindo força emocional, confiança e motivação, através da interação e experiências que permitem o aumento da energia interna. Um feedback positivo, para a PSC, transmite uma sensação de segurança em si mesma e de controlo da situação, sendo fortalecedor da sua autoestima e, consequentemente, motivação para o seu processo de recuperação (Lindberg et al.,2015; Wahlin, 2017).

Lindberg et al. (2015) defendem que a PSC deve ser envolvida no processo de capacitação e transição saúde-doença o mais precocemente possível. É importante para a PSC sentir-se incentivada para participar na relação e interação, mesmo quando não é capaz de o realizar autonomamente.

A parceria nos cuidados abrange a PSC, a sua família e o enfermeiro e é considerada como um dos fatores mais positivos e facilitadores da capacitação. O processo de capacitação requer compromisso combinado com relacionamentos nutritivos e interativos construídos na confiança e respeito mútuos. Os benefícios de melhorar a capacitação da PSC são extensos e são usufruídos não só pela PSC, mas por todos os elementos da tríade. Verifica-se uma diminuição do nível de angústia e tensão, maior coerência e controlo sobre a situação, desenvolvimento e crescimento pessoal (e/ou profissional), maior conforto, otimismo, satisfação interior e aumento da força emocional (Hardin, 2012; Wahlin, 2017).

A PSC sente-se mais capacitada quando é ouvida e envolvida ativamente no processo de tomada de decisão, considerando as suas preferências (como por exemplo quando é questionado se a PSC gostaria que lhe fosse retirado um dispositivo clínico). Desta forma, toda a experiência é facilitada e encorajada, contribuindo para o desenvolvimento da autodeterminação da PSC (Major et al., 2019; Wahlin, 2017). 
No que concerne ao comportamento dos profissionais de saúde, quando quem cuida demonstra humanidade e vontade de se conectar à pessoa doente, criando empatia, confortando e encorajando, é experienciada pela PSC uma sensação de segurança e pertença à comunidade (Lykkergaard \& Delmar, 2015; Wahlin, Samuelsson \& Agren, 2017).

A parceria nos cuidados é vivenciada em diferentes estados de dependência e independência e é orientada para o regresso da pessoa a uma condição de saúde, sendo a relação de empoderamento fonte de autenticidade, comunicação sensível e escuta ativa Lindberg et al., 2015; Wahlin, 2017).

A PSC considera que a família é fonte de inspiração e motivação para lutar e fortalecer o seu espírito de vida. Estes relacionamentos fortalecedores ajudam a PSC, família e enfermeiro a mobilizar recursos para lidar com os desafios existentes, por se sentirem acompanhados no seu percurso difícil. Falando concretamente no processo de capacitação, Meleis (2018) afirma que este é realizado através do processo de cuidados de enfermagem, no qual os enfermeiros descobrem as forças em saúde da pessoa doente e família e mobilizam as mesmas para apoiar os recursos já existentes, para que estas assumam a liderança ou presença ativa na sequencia de procedimentos.

Wahlin (2017) verifica que o conhecimento e o acesso a informações são ferramentas de poder e devem ser adaptadas às necessidades da PSC e família. Hardin (2012) afirma que estas ferramentas são úteis também para o enfermeiro, que poderá promover um cuidado individualizado se conhecer as necessidades especificas da pessoa doente, facilitando a gestão de sintomas.

Lykkergaard \& Delmar (2015) demonstram que a PSC valoriza quando as suas necessidades são atendidas, sem ter que pedir ajuda. A família, numa parceria de cuidados, é capaz de interpretar necessidades especificas e individualizadas da PSC. Esta capacidade é extremamente útil para o enfermeiro, que poderá decifrar o que alguns sinais significam para cada pessoa, para agir em conformidade (Hardin. 2012). Esta constatação é corroborada por Lusquiños, Mendes e Bento (2019, p. 1692), que numa revisão integrativa da literatura sobre o cuidado centrado na família, concluem que a família é um "recurso essencial e facilitador no cuidado ao doente" e que esta abordagem filosófica do cuidar deve ser coerente "por parte de toda a equipa multidisciplinar".

Em contexto de pandemia, apesar da restrição de visitas, surgiram algumas soluções para colmatar esta dificuldade. Quando a PSC já se encontra sem sedação (ou com sedação mais reduzida, estando desperta o suficiente), pode ser realizada comunicação com recurso às tecnologias e plataformas/ redes sociais. A família deve ainda ser incentivada a fornecer fotos de família, relatar momentos vividos que possam ser reproduzidos à PSC ou partilhar músicas do seu agrado (Devlin et al., 2020).

A própria família também deve ser capacitada desde cedo sobre o que pode esperar no futuro. Deve ser realizado um planeamento da alta em colaboração com a PSC e família, o qual permitirá que a família tome as providencias adequadas e desenvolva habilidades que a capacitem para a participação nos cuidados (Major et al., 2019; Wahlin, 2017).

$\mathrm{Na}$ Figura 2, é apresentado um esquema ilustrativo da interação entre PSC, família e Enfermeiro no processo de capacitação (tendo como influencia fatores facilitadores e inibidores), promovendo uma transição saúde-doença saudável.

Apesar da capacitação da PSC ter algumas condicionantes, por esta se encontrar num estado de maior fragilidade e, em algumas fases, não totalmente consciente, a autodeterminação é um dos atributos mais importantes neste processo (Wahlin, 2017). Este atributo é de extrema importância quando proveniente da PSC, mas também da familia e do enfermeiro, uma vez que esta tríade se deve unir em parceria ao longo de todo o processo de transição. 


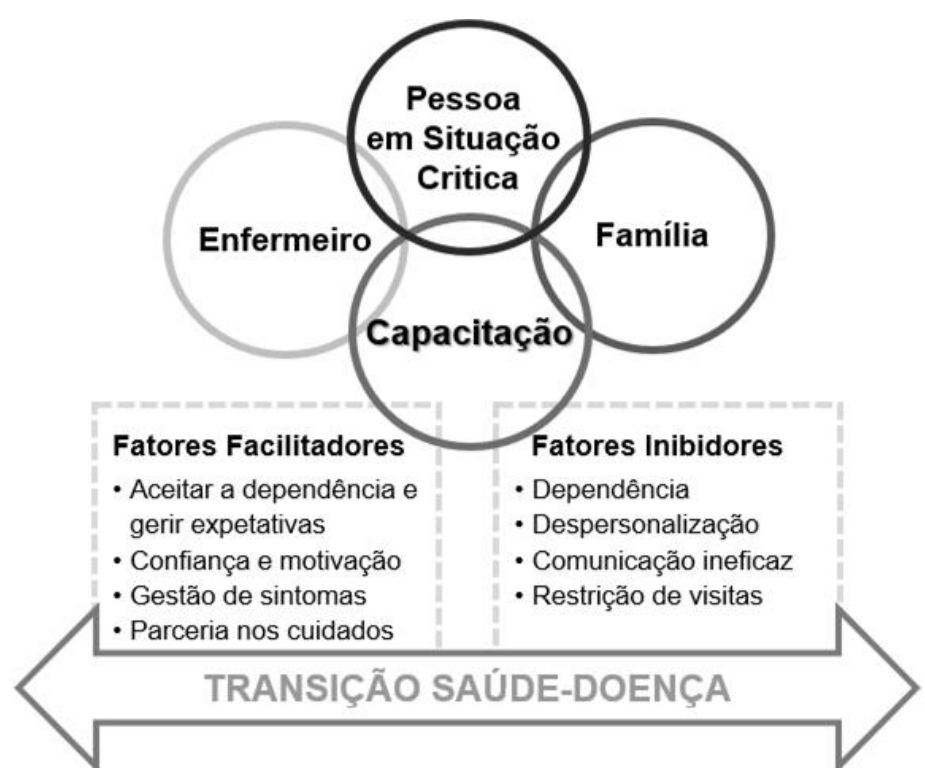

Fig. 2. Interação PSC, Familia e Enfermeiro no processo de Capacitação.

\section{Conclusões}

A capacitação da PSC é um processo que caminha lado-a-lado com a transição saúdedoença e tem alguns fatores facilitadores e inibidores. Como principais fatores inibidores surgiram: a dependência, a ausência de individualização nos cuidados, a comunicação ineficaz e a restrição de visitas. Como fatores facilitadores encontramos: aceitar a dependência e gerir expetativas; confiança e motivação; gestão de sintomas e parceria nos cuidados.

Nesta Revisão Integrativa da Literatura, em que emergiram predominantemente artigos de natureza qualitativa, foi possível concluir que a PSC, a família e o enfermeiro se devem unir em parceria para uma transição mais facilitada. A interação com os profissionais de saúde influencia a tomada de decisão do paciente e família e a construção de um processo de transição saudável.

Como Intervenções de Enfermagem que capacitam a PSC elencam-se: ajudar a pessoa a aceitar a dependência no autocuidado e a gerir expectativas; potenciar a confiança e motivação pela individualização dos cuidados e a gestão de sintomas; promover uma comunicação eficaz com pessoa doente e família, ao longo do processo de transição; subsidiar a gestão dos recursos disponíveis (pessoais, sociais e comunitários), que possibilitam o regresso ao quotidiano; assumir a família como parceira nos cuidados.

Conclui-se que as intervenções terapêuticas de enfermagem que capacitam a PSC, se enquadram num cuidado centrado no cliente, PSC e família.

A metodologia qualitativa possibilita, pelas narrativas conseguidas em entrevistas, o acesso à experiência vivida da pessoa doente, em que a transição saúde-doença é reveladora de enorme vulnerabilidade. Ao investigador, na produção e disseminação de resultados da pesquisa qualitativa, solicita-se a preocupação constante de traduzir o modo como a intervenção terapêutica de enfermagem pode fazer a diferença, assumindo-se como facilitadora no processo de transição saúde-doença. 


\section{Referências}

Benner, P., Kyriakidis, P. H., \& Stannard, D. (2011). Clinical wisdom and interventions in acute and critical care: A thinking-in-action approach (2ª Edição, Vol. 3). Estados Unidos da América: Springer publishing company

Devlin, J. W., O'Neal, H. R., Thomas, C., Barnes Daly, M. A., Stollings, J. L., Janz, D. R., Ely, E. W., \& Lin, J. C. (2020). Strategies to Optimize ICU Liberation (A to F) Bundle Performance in Critically III Adults With Coronavirus Disease 2019. Critical Care Explorations, 2(6), e0139. https://doi.org/10.1097/cce.0000000000000139

Funnell, M. M. (2016). Patient empowerment: What does it really mean? Patient Education and Counseling, 99(12), 1921-1922. https://doi.org/10.1016/j.pec.2016.10.010

Hardin, S. R. (2012). Engaging families to participate in care of older critical care patients. Critical Care Nurse, 32(3), 35-40. https://doi.org/10.4037/ccn2012407

Jones, S., Gill, P., \& Kenkre, J. (2020). Nurse managed patient focused assessment and care: A grounded theory of qualified nurses in acute and critical care settings assessing the mental capacity of adult patients. Journal of Clinical Nursing, 29(7-8), 1254-1266. https://doi.org/10.1111/jocn.15188

Lindberg, C., Sivberg, B., Willman, A., \& Fagerström, C. (2015). A trajectory towards partnership in care - Patient experiences of autonomy in intensive care: A qualitative study. Intensive and Critical Care Nursing, 31(5), 294-302. https://doi.org/10.1016/j.iccn.2015.04.003

Lusquiños, A., Mendes, A., \& Bento, M. (2019). O cuidado-centrado na Família da Pessoa em Situação Crítica na Unidade de Cuidados intensivos: Revisão integrativa da literatura. $O$ Congresso Ibero-Americano Em Investigação Qualitativa (CIAIQ), 2, 1685-1694. https://proceedings.ciaiq.org/index.php/CIAIQ2019/article/view/2400/2298

Lykkegaard, K., \& Delmar, C. (2015). Between violation and competent care - Lived experiences of dependency on care in the ICU. International Journal of Qualitative Studies on Health and Well-Being, 10, 1-10. https://doi.org/10.3402/qhw.v10.26603

Major, M. E., Van Nes, F., Ramaekers, S., Engelbert, R. H. H., \& Van Der Schaaf, M. (2019). Survivors of Critical Illness and Their Relatives A Qualitative Study on Hospital Discharge Experience. Annals of the American Thoracic Society, 16(11), 1405-1413. https://doi.org/10.1513/AnnalsATS.201902-1560C

Mamashli, L., Mohaddes Ardebili, F., Najafi Ghezeljeh, T., Manafi, F., \& Bozorgnejad, M. (2019). Investigating the Psychosocial Empowerment Interventions through Multimedia Education in Burn Patients. World Journal of Plastic Surgery, 8(3), 372-381. https://doi.org/10.29252/wjps.8.3.372

Meleis, A. I. (2010). Transition's theory: Middle-range and situation-specific theories in nursing research and practice. Springer Publishing Company.

Meleis, A. I. (2018). Theoretical nursing: development and progress (6th ed.). Wolters Kluwer.

Mendes, A. (2018). A interação enfermeiro-família na experiência vivida de doença critica: o cuidado centrado na família. Investigação Qualitativa Em Saúde, 2, 203-212.

Wåhlin, I. (2017). Empowerment in critical care - a concept analysis. Scandinavian Journal of Caring Sciences, 31(1), 164-174. https://doi.org/10.1111/scs.12331

Wåhlin, I., Samuelsson, P., \& Ågren, S. (2017). What do patients rate as most important when cared for in the ICU and how often is this met? - An empowerment questionnaire survey. Journal of Critical Care, 40, 83-90. https://doi.org/10.1016/j.jcrc.2017.03.004

Willis, D. G., Grace, P. J., and Roy, C. (2008). A central unifying focus for the discipline: Facilitating humanization, meaning, choice, quality of life, and healing in living and dying. Advances in Nursing Science, 31(1), E28-E40. 